\title{
Mouvements migratoires entre la Turquie et les Républiques turcophones du Caucase et d'Asie centrale
}

Les impacts religieux

Migratory movements between Turkey and the Turkish-speaking republics of

Caucasia and Central Asia. Religious impacts

\section{Bayram Balci}

\section{(2) OpenEdition}

1 Journals

\section{Édition électronique}

URL : http://journals.openedition.org/transcontinentales/795

DOI : 10.4000/transcontinentales.795

ISBN : : 978-2-7351-1557-0

ISSN : 1775-397X

\section{Éditeur}

Editions de la maison des sciences de l'homme

\section{Édition imprimée}

Date de publication : 31 décembre 2010

ISSN : 1950-1684

\section{Référence électronique}

Bayram Balci, « Mouvements migratoires entre la Turquie et les Républiques turcophones du Caucase et d'Asie centrale », Transcontinentales [En ligne], 8/9 | 2010, document 11, mis en ligne le 31 décembre 2010, consulté le 08 septembre 2020. URL : http://journals.openedition.org/transcontinentales/795 ; DOI : https://doi.org/10.4000/transcontinentales.795

Ce document a été généré automatiquement le 8 septembre 2020.

Tous droits réservés 


\title{
Mouvements migratoires entre la Turquie et les Républiques turcophones du Caucase et d'Asie centrale
}

\author{
Les impacts religieux \\ Migratory movements between Turkey and the Turkish-speaking republics of \\ Caucasia and Central Asia. Religious impacts
}

Bayram Balci

1 Toute étude sur la Turquie et l'aire turcophone, appelée aussi monde (ou espace ou sphère) turcique, nécessite au préalable d'en donner une définition. L'exercice est peu évident tant les concepts utilisés sont flous, polysémiques, et tendancieux puisque certains cachent des sensibilités politiques et idéologiques. Mais, partons du constat que, depuis le début des années 1990, la Turquie entretient des relations privilégiées avec des États issus de l'ex-URSs qui partagent avec elle un héritage historique et même géographique, l'Asie centrale étant considérée comme la patrie d'origine des Turcs avant leur migration en Anatolie par vagues successives de conquêtes ou de nomadisme. En effet, les Empires ottoman et seldjoukide qui ont vu naître la Turquie contemporaine furent fondés par des populations nomades projetées depuis leurs steppes centrasiatiques, à travers le plateau du Khorasan, avant de s'enraciner sur les terres anatoliennes.

2 Une bonne partie de la population turque actuelle possède des ascendances centrasiatiques, ce qui ne veut nullement dire qu'ethniquement tous les habitants de la Turquie contemporaine ont la même origine. Les populations anatoliennes et procheanatoliennes déjà sur place ou déportées par les conquérants turco-ottomans ont été mélangées avec les nouveaux venus et constituent la population de la Turquie actuelle. Mais, en dépit de ce brassage et bien que les Turcs de Turquie ne soient plus 
"purement turcs ", la référence aux racines centrasiatiques reste constante, malgré le puissant rêve européen qui anime ses élites comme le reste de la population.

\section{Considérations générales}

3 Pour entrer dans le vif du sujet, il s'agit pour nous d'analyser les relations et le phénomène migratoire entre la Turquie et plusieurs pays de l'ex-URss - Azerbaïdjan, Turkménistan, Ouzbékistan, Kirghizstan, Kazakhstan - mais aussi des pays limitrophes comme le Tadjikistan ou des régions autonomes de la Fédération de Russie, un vaste domaine géographique que les Turcs appellent monde turc, turk dunyasi, qu'on désigne en français par l'expression « monde turcophone » ou « turcique », et que la littérature anglo-américaine appelle Turkic ou Turkish World.

4 La Turquie partage avec ces États plusieurs points communs qui sont l'appartenance ethnique, la même origine géographique, la religion et, surtout, la langue même si des différences existent entre le turc anatolien et le kazakh, le kirghiz ou l'ouzbek. Depuis la fondation de la République turque par Mustafa Kemal, en 1923, jusqu'à la dislocation de l'URSS, en 1991, le discours en Turquie pour ces populations turciques était très peu élaboré et était souvent l'apanage de cercles intellectuels de droite, anticommunistes et aux relents parfois fascisants ${ }^{1}$. Cet intérêt pour les " Turcs de l'Extérieur » (Dis Turkler) était dans une très large mesure alimenté par des immigrés turciques (kazakhs, ouighours, ouzbeks, azéris) qui, fuyant le communisme, avaient trouvé refuge en Turquie ${ }^{2}$.

5 À partir de 1990, du fait de la dislocation de l'URss et de l'avènement sur ses cendres de républiques turciques ou turcophones indépendantes, ce discours à destination des Turcs de l'Extérieur a ressurgi et on a vu se développer un vaste intérêt pour ces nouveaux pays "frères", oubliés par la majeure partie de la population turque. Un contexte politique favorable - grâce au soutien des pays européens et des États-Unis pour que s'exerce une réelle influence turque dans la région supposée, à tort, proie facile de l'islamisme et de l'influence iranienne - et des autorités politiques centrasiatiques désemparées - car sommées soudainement de gérer une indépendance quasiment imposée - ont, parmi d'autres facteurs d'ordre géopolitique, éveillé en Turquie un rêve de grandeur, celui de se constituer et diriger un monde turc uni et solidaire, mais dont les contours n'ont jamais réellement été définis ${ }^{3}$. Ce contexte politique favorable, conjugué à l'esprit d'initiative des jeunes entrepreneurs anatoliens en quête de nouveaux marchés, a poussé des milliers de Turcs vers ces nouvelles républiques. Très rapidement, un vrai phénomène migratoire s'est développé entre la Turquie et ces républiques, porteur et vecteur de plusieurs discours idéologiques où le religieux occupe une place non négligeable.

\section{Tentative de description du phénomène migratoire turc en Asie centrale}

6 Il est difficile d'établir un panorama précis et détaillé, quantitativement et qualitativement, du phénomène migratoire anatolien en Asie centrale et dans le Caucase, à savoir principalement en Azerbaïdjan, Turkménistan, Kazakhstan, Ouzbékistan, Kirghizstan et Tadjikistan. Les données statistiques étant quasi 
inexistantes, on ne peut fournir qu'une esquisse générale du phénomène. Il s'agit généralement de petits commerçants, restaurateurs et responsables de petites et moyennes entreprises, très souvent dans le bâtiment et la construction mais aussi dans l'agroalimentaire et la petite industrie. On trouve également, dans une moindre mesure, des entreprises turques de taille internationale dans le domaine de la construction et du textile, voire même dans celui des hydrocarbures, ainsi la compagnie nationale turque de pétrole, TPAo, très active en Azerbaïdjan où elle est associée à la gestion du pétrole de la Caspienne, notamment pour son acheminement avec le fameux pipeline Baku-Tbilissi-Ceyhan.

7 Les statistiques font défaut sur le nombre exact de migrants turcs en Azerbaïdjan, d'abord à cause du manque de services publics de part et d'autre affectés précisément à la question migratoire entre les deux aires, mais aussi du fait du grand nombre de migrants turcs y travaillant sans enregistrement officiel et y résidant sans aucun titre de séjour légal. Toutefois des estimations permettent d'avancer le chiffre de 50000 Turcs en Azerbaïdjan, principalement à Bakou et dans son agglomération proche. Au Kazakhstan, la présence migratoire turque, beaucoup plus massive, dépasse les 100000 personnes. L'économie locale est très dynamique et attire des travailleurs et des commerçants non seulement dans les deux capitales, Astana et Almaty, mais aussi dans les autres villes du pays, comme à Chimkent où se trouve une université turcokazakhe, et encore dans des villes pétrolières comme Atirau et Aktau sur la Caspienne. Les nombreuses entreprises turques importent parfois leur main-d'œuvre adaptée et qualifiée de Turquie, ce qui entraîne des frictions entre immigrés turcs et travailleurs kazakhs. Au Turkménistan, dont la capitale Achkhabad est en chantier permanent depuis au moins quinze ans, les entreprises turques de BTP, concurrentes directes du groupe français Bouygues, font venir des travailleurs de Turquie. Leur nombre global avoisine les 20000 , chiffre non négligeable si l'on compte que la population du pays ne dépasse pas les 4 millions d'habitants. Au Kirghizstan, la présence migratoire turque est tout aussi visible. En Ouzbékistan, en revanche, les relations politiques complexes avec Ankara ont des effets réducteurs directs sur les mobilités entre les deux pays.

Le phénomène migratoire s'effectue aussi dans l'autre sens, depuis les républiques turcophones vers la Turquie. Au départ, ces migrants étaient des enseignants et des universitaires notamment, des gens qualifiés mais dont le niveau de vie a périclité de façon soudaine à la fin de l'Union soviétique. Aujourd'hui, la migration depuis le centre de l'Asie vers la Turquie devient de moins en moins « intellectuelle » et ce sont surtout des commerçants ou de la main-d'œuvre pour alimenter les chantiers turcs qui se déplacent. Par ailleurs, une caractéristique essentielle de cette migration est son aspect féminin, liée au développement de plusieurs réseaux de prostitution entre la Turquie et l'Asie centrale. Cette activité est difficile à quantifier mais son importance est soulignée par de nombreuses associations qui étudient le phénomène. Enfin, on ne saurait oublier la présence de plusieurs milliers d'étudiants originaires de ces Républiques, inscrits dans les universités turques avec souvent le soutien financier du gouvernement d'Ankara. Il y aurait à l'heure actuelle, en Turquie, entre 2000 et 3000 étudiants de chaque république, à l'exception de l'Ouzbékistan qui freine ses relations avec la Turquie $^{4}$. 


\section{Les flux idéologiques véhiculés par cette migration à double sens}

9 Le lien entre migrations, migrants et diffusion d'idées religieuses est une réalité bien connue qui a souvent été étudiée. En cela, notre recherche n'a rien d'exceptionnel mais contribue de sa pierre à l'édifice général. Ce lien entre migration et circulation d'idées religieuses est par ailleurs complexe, dynamique et interactif. L'immigration peut induire indirectement un message religieux, et, inversement, le discours religieux peut être la raison même de la migration, c'est-à-dire le résultat de la volonté missionnaire et de pèlerinage du candidat à la migration. Ne dit-on pas que le Prophète Mohammad a immigré de la Mecque à Médine pour exporter sa foi et la diffuser par la suite dans la péninsule Arabique et au-delà ? Cette remarque vaut pour toutes les idées, religieuses ou autres. Par ailleurs, le message véhiculé peut être en cours de route altéré et prendre des formes inhabituelles, hybrides.

10 Dans le cas des relations migratoires entre la Turquie et l'Asie centrale, on observe le même phénomène. Plusieurs courants idéologiques ont été provoqués ou ont provoqué un mouvement migratoire porteur de message idéologico-religieux. Le premier discours ainsi véhiculé a été nationaliste voire racial. Cependant, il n'a pas obtenu tout le résultat escompté, ce qui nous permet de l'analyser plus rapidement. Ensuite, nous examinerons le principal discours idéologique, le discours islamiste, et ses différents courants partis de Turquie dans les valises des migrants turcs, pour souder ou ressouder cette vaste Asie centrale turcophone à la Turquie.

11 Jusqu'à la chute de l'Union soviétique, les courants nationalistes turquistes, de tendance droite réactionnaire, étaient les seuls en Turquie à s'adresser et à s'intéresser aux les populations turciques d'URss. Leur discours prônait la grandeur de la race turque, et ils vouaient un véritable culte au foyer originel des Turcs, l'Asie centrale et la Sibérie, appelé Touran dans la phraséologie nationaliste. Ce courant, au demeurant très hétéroclite et traversé par des clivages de diverses natures, a souvent été animé par des réfugiés azéris, kazakhs, ouzbeks, tatars, ouighours ayant fui le communisme soviétique ou chinois et établis en Turquie. Ils y créaient des associations, des revues et des clubs où la référence à ce Turkestan perdu est constante 5 . Ces courants nostalgiques de la Patrie perdue rêvaient de la dislocation de l'Union soviétique qui permettrait à leur pays de retrouver l'indépendance et la liberté.

$12 \mathrm{Au}$ moment où l'Union soviétique s'effondre, les partisans de ces courants crient victoire. Ils estiment proche le moment où leur rêve deviendra réalité avec la création d'un monde turc uni allant de la Turquie jusqu'à la frontière chinoise. Certains individus migrent même dans ces pays "enfin libérés du communisme". Ils sont encouragés par un contexte géopolitique international favorable et des autorités politiques qui multiplient les déclarations de retrouvailles entre frères turcs, dans un même monde qui va, selon le président de l'époque, Süleyman Demirel, «de l'Adriatique à la Muraille de Chine ».

Or, ce rêve s'est vite heurté à la dure réalité du terrain, à savoir la surdité des " frères » turcs à ce discours panturquiste. Ces migrants, partis faire du commerce ou des études dans ces pays voisins ont constaté avec amertume le décalage cinglant entre leurs rêves et la réalité : différences de culture, de mentalité et même de langue entre ces peuples qu'ils croyaient pourtant frères. Tirant la conclusion de l'inexistence d'un monde turc, 
la plupart ont renoncé à leur idéologie et ont appris le russe, langue de communication par excellence de la région.

Une exception mérite tout de même d'être mentionnée. En Azerbaïdjan, ce discours de retrouvailles n'a pas complètement échoué. Plus que les autres peuples turcophones, les Azéris - la société comme le pouvoir - revendiquent cette fraternité avec les autres peuples turcophones mais surtout avec la Turquie. Pour des raisons géopolitiques complexes notamment liées à la question arménienne, ils ont une vision de la politique régionale convergente avec la Turquie. À l'heure actuelle, dans les rues de Bakou comme dans les bureaux officiels, on entend très régulièrement, pour qualifier les relations turco-azéries, le slogan «Une même nation, deux États ». Dans ce cas précis, les migrants ont été les porteurs du discours panturquiste, en même temps que ce rêve les a soutenus dans leur choix de migration, même si les considérations économiques ont été aussi très présentes.

\section{L'essentiel, les courants religieux au centre de cette dynamique migratoire}

Plus que les courants nationalistes, ce sont les mouvements islamistes qui ont le plus bénéficié de l'établissement des liens avec la Turquie. Se sont déversées dans la vaste Asie centrale, dans le Caucase et jusque dans les régions musulmanes de la Fédération de Russie, plusieurs vagues successives de missionnaires anatoliens venus islamiser ou ré-islamiser ces terres fraîchement libérées de l'idéologie communiste et de la propagande soviétique antireligieuse. Pour la plupart, des groupes privés, et en principe indépendants des acteurs étatiques, investissent le champ religieux. Mais, au préalable, il s'impose d'expliquer l'attitude des États face à l'islam en cette période de fortes turbulences pour tous les régimes issus de l'ex-URss. Car, même si les flux islamiques entre la Turquie et l'Asie centrale ont été l'œuvre de mouvements privés et fortement liés au phénomène migratoire, les acteurs étatiques n'ont pas été indifférents et leurs perceptions de la situation à l'époque n'a pas été sans effet sur les résultats des actions missionnaires privées déployées dans ces pays.

Pour la Turquie de ce début des années 1990, comme il a été souligné précédemment, l'espace turcophone du Caucase et d'Asie centrale représente un nouvel espace d'investissements en termes politiques, économiques et culturels. Outre la mise en place d'un vaste programme éducatif qui permet à des milliers de jeunes étudiants turcs d'étudier en Asie centrale et à des milliers de jeunes centrasiatiques de poursuivre des études en Turquie ${ }^{6}$, l'État turc a aussi, ce qui est audacieux pour une république laïque et séculière, mis en œuvre un véritable mécanisme de coopération islamique avec ces États, principalement par le biais de ses ambassades où est affecté un attaché aux affaires religieuses chargé $\mathrm{d}^{\prime}$ ' exporter " la vision officielle turque de l'islam et de sa place dans la société. Cet attaché, par exemple, coordonne les programmes d'échanges avec la Turquie en matière de formation de nouveaux imams et de restauration ou de construction de mosquées. Dans certains cas, il a aussi prise sur les activités religieuses déployées par les migrants turcs dans le pays d'accueil.

Pour les États nouvellement indépendants, la question du contrôle des flux religieux ne se pose pas dans un premier temps. Bien que crispées et anxieuses face à une indépendance davantage imposée depuis Moscou lors de la chute de l'Empire 
soviétique, les autorités politiques, toutes issues de l'ancien système, se complaisent tout de même dans l'euphorie illusoire de l'ouverture au monde et de l'établissement de contacts avec des États et des peuples longtemps tenus à distance par le pouvoir central soviétique ${ }^{7}$. Dans ce contexte, la circulation des idées et des hommes se développe dans un enthousiasme positif partagé par tous. Des contacts avec l'extérieur sont établis sur la base de solidarités ethnique et/ou religieuse. Un exemple assez révélateur qui relève à la fois de la question migratoire et du phénomène religieux n'est autre que le hadj, le pèlerinage à la Mecque. Très limité pendant la période soviétique, il connaît une forte croissance dès 1992. Il est organisé librement par des individus ou des sociétés privées mais très rapidement repris en main par les États qui souhaitent mieux contrôler le développement de la religiosité.

Dans ce cadre au départ bon enfant d'ouverture vers l'extérieur, s'opère la rencontre entre les idées religieuses, transportées par des migrants originaires de différents pays, et une population locale avide de contact avec un étranger proche par la culture, la religion et la langue. La Turquie, à la fois semblable et différente, devient une source d'inspiration et d'importation d'idées religieuses.

Dès cette période où les liens migratoires commencent à s'établir entre la Turquie et l'Asie centrale, plusieurs mouvements, organisations, tendances, ou phénomènes confrériques ou néoconfrériques turcs s'intéressent alors à cette région du monde. Nous nous attarderons plus particulièrement sur quatre d'entres eux : le mouvement de Sait Nursi dont les disciples sont appelés nurcu; les enseignants éducateurs de Fethullah Gülen qui est un des héritiers spirituels de Sait Nursi; les disciples de Suleyman Tunahan, une grande figure islamique qui a marqué la Turquie des années 1970 et dont les disciples, nombreux en Asie centrale, sont communément appelés les suleymanchis; des sous-groupes de la grande confrérie nakshibendie qui, à partir de leur base arrière d'Istanbul, ont développé un vaste programme d'assistance humanitaire teinté d'intentions islamiques dans tout l'espace musulman de l'ex-URSS, principalement en Asie centrale. Les frontières entre ces mouvements sont souvent mouvantes et des passages peuvent être observés d'un groupe à l'autre, surtout quand les besoins économiques ou les opportunités d'expatriation les rendent nécessaires.

Les nurcufurent parmi les premiers à saisir l'opportunité offerte par l'ouverture des frontières de l'ex-URSs pour s'exporter en Asie centrale. Le terme nurcu renvoie à Sait Nursi, une grande figure de l'islam turc née à l'est de la Turquie (1876-1960) et qui occupa une place essentielle dans l'histoire religieuse de la Turquie moderne ${ }^{8}$. Émergeant dans un contexte de fin d'empire, ce mouvement est avant tout piétiste et cherche à lutter contre la perte de la foi et de la pratique au sein de la population turque de l'époque, très marquée par les réformes séculières de Kemal Atatürk. Conservatrice mais à la fois nationaliste et islamiste modérée, cette organisation n'est pas politique mais cherche à placer l'islam au centre de la société turque et dans l'espace public. Interdite et condamnée par le régime kémaliste, mais tolérée lors du passage à un système politique plus démocratique, notamment dans les années 1950, elle a toujours oscillé entre bannissement et existence légale. À la mort du père fondateur, le mouvement se scinde en plusieurs tendances, mais toutes sont caractérisées par une pratique militante qui cherche à desserrer le contrôle excessif de l'État turc sur la religion.

21 Au début des années 1990, différentes tendances issues de ce mouvement s'exportent en Asie centrale, très souvent par le biais du petit commerce et du travail dans les 
grandes entreprises turques de construction. Le discours religieux de leurs membres n'a rien de révolutionnaire. La plupart du temps leur action se limite à organiser, dans des madrasas semi-officielles ou simplement dans des maisons ou appartements collectifs, des lectures de l'œuvre maîtresse de leur père spirituel, la Risale $i$ Nur. Ce texte, «Épitre de la Lumière ", n'est autre qu'une exégèse du Coran écrite dans une langue plus ottomane que turque, à tel point que, sans un dictionnaire ou l'assistance d'un maître, sa lecture ou sa compréhension se trouvent quasiment impossibles. Semilégales, les activités de ces migrants turcs sont, selon les pays, sous étroite surveillance. Le mouvement comptait énormément sur les possibilités d'action prosélyte en Ouzbékistan, pays le plus peuplé et le plus musulman de la région. Or, les relations diplomatiques tendues entre Ankara et Tachkent et surtout le manque flagrant de libertés religieuses ont considérablement freiné son implantation dans ce pays. Dans les autres républiques d'Asie centrale, le mouvement continue d'être véhiculé par les migrants turcs, mais aussi de plus en plus par des adeptes locaux, enrôlés grâce à une action prosélyte engagée depuis vingt ans.

Issu de ce même mouvement fondé par Sait Nursi, l'organisation de FethullahGülen, un gourou à la mode anatolienne, mérite une place particulière dans l'analyse tant son action et son impact sont considérables dans l'exportation d'un islam turc en Asie centrale et dans le Caucase. Le mouvement, doté d'une identité à géométrie variable, tantôt néo-confrérie, tantôt mouvance ou même nébuleuse, est fortement lié à la personnalité de son fondateur. Cette mouvance religieuse aux contours imprécis s'est formée sur une longue période, entre les années 1970 où, dans la région d'Izmir, Gülen et ses proches créèrent plusieurs associations culturelles et éducatives, et les années 1990, caractérisées en Turquie par une remarquable entrée dans la libéralisation économique permettant l'émergence de centaines d'entreprises économiques très fortement structurées autour de valeurs islamiques'. À partir d'un noyau d'associations rassemblant des parents d'élèves et des éducateurs, la mouvance gagne la confiance de milliers d'entreprises et d'entrepreneurs et parvient à bâtir un pseudo « holding » sans personnalité juridique distincte et précise mais disposant d'un puissant levier économique et financier dépassant les frontières de la Turquie.

Le contenu idéologique de la tendance est difficilement identifiable, car Fethullah Gülen se veut apolitique et ses prédications et discours empruntent à des registres très variés, mystique soufie, nationalisme turc, islam progressiste et moderniste, œcuménisme islamo-chrétien entre autres. À la fin des années 1980, le mouvement est à son apogée et commence même à inquiéter les gardiens du dogme kémaliste. L'armée et les milieux séculiers craignent qu'il ne se transforme en force politique après une longue période de gestation dans un cadre éducatif et associatif.

24 Cette fin de décennie est aussi celle de l'établissement de liens divers entre la Turquie et l'ex-URss, une aubaine pour les entreprises proches de Gülen désormais trop puissantes pour se limiter au territoire proprement turc et à la recherche de nouveaux marchés commerciaux et de nouvelles terres de prédication.

25 C'est ainsi que des petits commerçants, des travailleurs, des éducateurs et parfois même des entreprises de taille internationale, se trouvant plus ou moins dans la sphère spirituelle de Fethullah Gülen, se sont exportés en Asie centrale pour y travailler et propager par la même occasion les idées de leur maître ${ }^{10}$. Ces disciples de Gülen, les fethullahci, s'illustrent notamment par la création et l'animation de tout un réseau éducatif de lycées et de centres de langue, voire même de centres de préparation aux 
concours universitaires. L'enseignement y est séculier et laïc en toute conformité avec les normes nationales héritées de la période soviétique ; aucun enseignement coranique ou islamique n'est dispensé dans les lycées et aucune littérature islamique n'y est propagée. Mais, et c'est là toute la subtilité de leur méthode, sans cours de religion dans des écoles prétendument laïques, le mouvement parvient tout de même à véhiculer la pensée de Fethullah Gülen, prônant un islam à la fois turc national, en bons termes avec le gouvernement en place, marqué par le soufisme et dépourvu de toute ambition politique $^{11}$. Dans chaque pays d'Asie centrale, les dizaines d'écoles fondées par le mouvement travaillent en symbiose avec des entreprises turques implantées dans le pays. Le corps enseignant est constitué de jeunes professeurs, tout juste sortis des universités turques, partis en Asie centrale pour des motivations à la fois économiques et spirituelles, et pour diffuser une forme évoluée d'islam dans une région considérée comme le pays d'origine, celui de leurs ancêtres. Comparables, toute proportion gardée, aux jésuites partis d'Europe occidentale pour propager la bonne parole chrétienne à travers le monde, les cadres supérieurs de Gülen avouent volontiers s'inspirer de ce phénomène missionnaire pour, à leur tour, exporter leur foi et leur culture dans de nouvelles contrées.

Un autre mouvement turc, moins structuré et moins riche, mais avec la même logique de dissémination de migrants anatoliens en Asie centrale et dans le Caucase, tente à sa manière de contribuer au flux spirituel qui, à partir de la Turquie, vient irriguer les friches postsoviétiques. Il s'agit des disciples d'un autre grand intellectuel musulman turc, Hilmi Suleyman Tunahan ${ }^{12}$, appelés les suleymanci, qui diffusent, plus ouvertement que les fethullahci, la philosophie islamique de leur maître. Du temps de son fondateur, la communauté s'était faite remarquer par ses querelles constantes avec la Diyanet, la plus haute instance officielle de gestion de l'islam turc, qui voulait imposer son monopole, ce que refusaient certains mouvements privés dont celui de Suleyman Tunahan. Le mot d'ordre des suleymanci a toujours été " permettre à chaque musulman de pouvoir lire le Coran en arabe ». Leitmotiv qui n'a guère changé puisque, en Turquie comme dans leur pays d'expatriation, ces militants placent au centre de leurs activités et de leurs préoccupations la fondation d'écoles coraniques, dont la priorité absolue est de permettre aux jeunes générations l'accès au Coran en langue arabe. L'islam promu par ces expatriés turcs véhicule aussi une certaine idéologie turquiste, du moins met en avant une image positive de la Turquie. Dans chaque pays où le mouvement est implanté, il entretient de bonnes relations avec les instances officielles de l'islam et les organisations islamiques modérées. Comme d'autres organisations islamistes turcs, il s'est au départ appuyé sur la migration turque pour s'exporter en Asie centrale. Toutefois, à l'heure actuelle, il a formé sur place suffisamment de cadres locaux, kirghizes et kazakhs notamment, pour constituer de nouvelles élites islamiques.

Autant que les autres, cette mouvance s'appuie sur les commerçants et petits entrepreneurs turcs pour se développer à l'étranger. Elle a ouvert dans tous les pays de la région, sauf en Ouzbékistan et au Tadjikistan, de modestes madrasas entièrement financées par des fondations et associations implantées en Turquie et des entreprises turques présentes en Asie Centrale et dans le Caucase. Régulièrement des nouveaux cadres religieux arrivent de Turquie et certains jeunes étudiants sont envoyés dans les fondations privées de la nébuleuse suleymancie partout en Turquie pour y perfectionner leur formation. Cette organisation s'est surtout implantée dans la région caucasienne, en Azerbaïdjan et en Géorgie, notamment dans la province d'Adjarie. 
Particularité de sa présence dans cette région, elle utilise les descendants de la diaspora caucasienne en Turquie pour diffuser ses idées. En effet, de très nombreuses associations regroupent les descendants de réfugiés caucasiens venus en Turquie pour fuir la domination russe.

Depuis la fin de l'Urss, une partie des échanges, de tout genre, entre la Turquie et le Caucase est le fait de ces enfants de la diaspora. Ainsi, une bonne partie des missionnaires envoyés dans ces contrées ont des origines caucasiennes.

Les nakshibendis constituent le quatrième et dernier groupe turc important à s'être très intéressé à l'Asie centrale. Ainsi, certaines de leurs organisations ont développé, en s'appuyant sur un grand nombre de volontaires turcs au départ, plusieurs activités pour renouer le contact avec les nakshibendis de l'ex-URss et pour apporter leur contribution au processus de réislamisation de cette région. La nakshibendiyya, du nom de Bahaduddin Nakshibendi, un mystique du $\mathrm{xv}^{\mathrm{e}}$ siècle né à dix kilomètres de Boukhara, est une confrérie soufie qui, à partir de ce foyer centrasiatique, s'est diffusée dans tout le monde musulman, principalement en Inde, dans le Caucase et en Turquie ${ }^{13}$. Prônant un islam mystique modéré mais plus ou moins politisé selon les époques et les lieux, cette confrérie occupe une place essentielle dans le champ islamique de Turquie où elle est incarnée par plusieurs branches animées par d'importantes autorités religieuses. Dans leur ensemble, quelle que soit leur branche, les nakshibendis de Turquie ont toujours entretenu une forte nostalgie pour la patrie du fondateur de l'ordre et l'on sait qu'au début des années 1990, Turgut Ozal, Premier ministre et par la suite président de la République, par admiration pour cet ordre qui lui avait apporté un soutien politique et électoraliste non négligeable, a généreusement contribué à la restauration du mausolée et facilité les premiers contacts entre les Nakshibendis de Turquie et leurs confrères d'Ouzbékistan ${ }^{14}$.

Forts de ces soutiens, les disciples de cette confrérie ont également utilisé le flux important des travailleurs turcs en Asie centrale pour mettre leur entreprise mystique en contact avec la patrie de la nakshibendiyya, l'Ouzbékistan, où se trouve le mausolée $\mathrm{du}$ fondateur cette confrérie, Bahaduddin Nakshibend. Mais, surtout, le pèlerinage sur la tombe de Bahaduddin Nakshibend à Boukhara a permis de recréer des liens entre ses disciples de Turquie, d'Asie centrale et d'ailleurs. Un véritable tourisme religieux, impensable pendant la période soviétique, s'est développé à partir de la Turquie d'où des compagnies proposaient, dès 1990, un parcours incluant Tachkent, Boukhara, Ghijduvan et Samarkand, où se trouvent d'importants sites liés à la nakshibendiyya.

31 Paradoxalement, c'est moins dans leur patrie originelle, en Asie centrale, que dans les villes du Caucase, en Azerbaïdjan et en Géorgie notamment, que les nakshibendis turcs ont été les plus influents et les plus à même de proposer leurs «services»: un enseignement islamique en langue turque, des œuvres caritatives, la restauration ou reconstruction de certaines mosquées ayant souffert par le manque d'entretien pendant la période soviétique, etc. En Azerbaïdjan, plus massivement dans les régions sunnites que chiites, la branche nakshibendie, liée à la fondation Mahmut Hudayi, est animée à l'heure actuelle par le leader nakshibendi Osman Nuri Topbas, originaire de la ville de Uskudar-Istanbul. Cette branche gère une demi douzaine d'établissements religieux dans lesquels se forment les nouvelles élites musulmanes du pays ${ }^{15}$. Comme dans le cas des autres mouvements, d'importants échanges et de va-et-vient ont lieu entre la Turquie et le pays d'implantation, qui favorisent le transfert de savoir dans les deux sens. Car, si les Turcs cherchent à exporter leur vision de l'islam, ils reçoivent en 
retour, dans une moindre mesure certes, des influences islamiques issues des traditions de ces pays-là et, plus particulièrement d'Asie centrale, d'où sont originaires nombre d'étudiants venus en Turquie pour leurs études universitaires.

\section{Comment mesurer l'impact de ces flux religieux sur les sociétés d'Asie centrale et du Caucase?}

Bien que déployé sur une courte période, de 1991 à nos jours, le phénomène migratoire en Asie centrale et dans le Caucase a particulièrement affecté l'espace urbain et les sociétés locales. La présence turque expatriée ou immigrée, dans la sphère économique, industrielle, commerciale ou bien culturelle, éducative et religieuse est particulièrement visible et fait partie des réalités quotidiennes des grandes villes de Bakou à Almaty en passant par Achkhabad, Tachkent, Bichkek et Astana. L'augmentation du nombre des mariages mixtes favorise un certain brassage et accentue le phénomène migratoire définitif de ceux qui au départ n'étaient qu'expatriés. Même si en termes de gros capitaux la présence économique turque est plus faible que celle des grandes puissances économiques mondiales comme l'Allemagne ou les États-Unis, les entreprises étrangères de taille moyenne sont très souvent originaires de Turquie. Sans même que l'enseignement du turc soit particulièrement présent dans les écoles et les universités, le turc anatolien est connu voire pratiqué par de plus en plus de gens, grâce à la forte et constante présence turque depuis plus de quinze ans. La proximité linguistique entre le turc anatolien et les langues turciques locales facilite aux Turcs l'apprentissage de l'ouzbek, du kazakh, mais inconsciemment ou consciemment c'est le turc qu'ils imposent à leurs partenaires locaux. Dans les grandes entreprises turques en tout cas, la langue de travail est le turc, devant la langue officielle locale (kazakhe, ouzbek, kirghize, turkmène..) et le russe.

En termes religieux, l'impact de la migration turque en Asie centrale est réel, bien que difficile à évaluer avec précision; il est souvent tributaire de la nature des relations entre la Turquie et chacun des États de la région. De même, le degré de liberté qui prévaut dans chaque pays, notamment dans le domaine religieux, détermine l'importance de l'influence des missionnaires turcs en Asie centrale.

Dans un souci de simplicité, procédons à un bilan par pays. Dans l'État le plus proche, à tous points de vue, de la Turquie, l'Azerbaïdjan, les migrants turcs ont été parmi les premiers à s'implanter, dès 1990, dans certaines villes, avant même les religieux iraniens pourtant très proches. En convergence avec l'action de l'État turc qui leur a facilité la tâche, et pour une série de raisons à la fois politiques et géopolitiques (la question arménienne notamment), les migrants turcs affiliés à l'une ou l'autre des organisations religieuses évoquées ci-dessus ont déjà formé des centaines de membres des nouvelles élites religieuses à travers tout le pays. Au sein même de l'appareil de gestion de l'islam, de la direction des affaires religieuses mais aussi du comité d'État pour les affaires religieuses, on trouve de nombreux cadres formés sur place par les fondations caritatives turques. En cela, les organismes privés originaires d'un pays laïc et séculier comme la Turquie ont été plus performants que les missionnaires envoyés par la République islamique d'Iran. Cela montre à quel point le contexte politique est important, y compris quand il s'agit de la circulation des idées religieuses.

Le Turkménistan est aussi un cas assez intéressant dans cette analyse des flux religieux turcs en Asie centrale. Malgré la fermeture de son régime et son manque de libertés 
religieuses, le Turkménistan a été un terrain de prédication particulièrement bien investi par les mouvements islamiques turcs mais aussi par l'État turc. En effet, l'attaché aux affaires religieuses de l'ambassade de Turquie gère depuis plus de quinze ans de vrais programmes de coopération islamique, notamment de formation, grâce à la création d'une faculté de théologie. Une visite dans la plupart des villes de ce pays avec, à chaque fois, des haltes importantes dans les principales mosquées, nous a permis de mesurer l'importance de cet impact turc sur l'islam turkmène. La plupart des imams formés depuis l'indépendance du pays ont un rapport direct avec la Turquie : ils ont été formés soit par l'État turc, soit par des fondations privées qui déploient leurs activités dans le pays d'une manière parfois illégale d'ailleurs. Le mouvement migratoire entre la Turquie et le Turkménistan a joué un rôle majeur dans ces échanges religieux. Des étudiants turcs en grand nombre, en plus des enseignants des écoles turques de la mouvance de Gülen, ont pu travailler dans la plupart des villes importantes du Turkménistan. De la même manière, des milliers d'étudiants turkmènes ont séjourné en Turquie depuis l'indépendance de leur pays, séjour au cours duquel ils ont été en contact avec l'islam turc qu'ils ont en partie importé dans leur pays.

L'Ouzbékistan est un cas plus complexe à analyser, tant ses relations avec la Turquie se sont dégradées depuis le milieu des années 1990, après avoir été si chaleureuses et cordiales durant les premières années de l'indépendance ouzbèke. Pour les résumer à l'extrême, les relations ouzbeko-turques ont souffert lorsque, en 1994, les partis Erk et Birlik, opposés au régime de Tachkent, se sont installés en Turquie où ils ont bénéficié du soutien de certains milieux nationalistes turcs, sans parvenir toutefois à gagner la confiance des autorités officielles turques. En revanche, ils étaient particulièrement populaires parmi les milliers d'étudiants ouzbeks envoyés par Tachkent en Turquie si bien que, rapidement, le régime de Karimov, qui a toujours été suspicieux à l'égard de toute idée subversive, a fait rapatrier la quasi-totalité des étudiants ouzbeks et gelé ses relations avec la Turquie.

En dépit de la méfiance des Ouzbeks qui ont toujours étroitement surveillé toute influence extérieure, certains mouvements islamiques turcs se sont bien implantés dans les principales villes ouzbèkes tout au long des années 1990. Le mouvement de Gülen était présent massivement dans tout le pays. Mais, à partir de septembre 2001, le pays sombrant dans la paranoïa a mené une véritable chasse aux sorcières contre les Turcs, accusés de subversion. Toutes les écoles ont été fermées du jour au lendemain ; certains entrepreneurs liés à ces écoles ont été expulsés, et des Ouzbeks compromis avec ces établissements ont écopé des peines de prison. Cette épuration n'a pas complètement réussi à éradiquer les idées déjà véhiculées par ces missionnaires turcs puisque, en avril 2009, plusieurs membres ouzbeks soupçonnés d'appartenir à la mouvance nurcue et de propager leurs idées subversives ont été arrêtés et mis en prison $^{16}$.

Le Kirghizstan, mais surtout le Kazakhstan avec ses pharaoniques projets de construction dopés par la manne pétrolière, attirent de nombreux entrepreneurs étrangers. Parmi eux, des Turcs originaires de la Turquie profonde, cette Anatolie moralement conservatrice mais économiquement très dynamique. Les mouvements islamistes turcs évoqués, les nurcus, les fethullahcis et les autres, y trouvent un terrain particulièrement propice à leurs activités. Ils sont en cela indirectement encouragés par les autorités kazakhes qui y voient un moyen de favoriser le renouveau de la culture kazakhe dont les Turcs se font les porte-parole sur place, dans un contexte 
démographique où l'élément slave est encore très important (environ $40 \%$ de la population). Pour les autorités kazakhes, la présence turque fait contrepoids à la forte composante slave de la population. Pour les missionnaires turcs, avec une université à Almaty, trente-cinq établissements de niveau lycée répartis dans tout le pays et une vingtaine de modestes madrasas gérées par les suleymancis, le Kazakhstan est une terre de conquête, un vaste territoire vierge, peuplé autant de Russes et que de Kazakhs, tous très perméables à la propagande religieuse qui y faisait défaut depuis longtemps. Aujourd'hui, au Kirghizstan comme au Kazakhstan, tous les cadres religieux sont redevables d'une manière ou d'une autre aux autorités religieuses turques ou à ces mouvements religieux turcs confrériques privés. Ainsi, l'islam turc sous toutes ses formes exerce une influence considérable sur l'establishment religieux local, voire même sur les nouvelles élites politiques souvent tentées de s'inspirer de la Turquie pour bâtir une nouvelle nation où le facteur religieux n'est pas totalement marginalisé.

L'islam ou plutôt les islams de Turquie ont une aura bien plus large et leur influence dépasse le cadre des républiques turcophones puisque le Tadjikistan, les régions turcophones de la Fédération de Russie, notamment le Tatarstan et le Bachkortostan, sont également des terrains importants pour leur action missionnaire appuyée par le soutien des entreprises et travailleurs turcs.

\section{Conclusion}

Pour une multitude de raisons, politiques, économiques, identitaires, sécuritaires, aucun État moderne ne peut rester indifférent face au phénomène migratoire, qu'il s'agisse d'immigration ou d'émigration, d'entrée sur son territoire de citoyens étrangers ou de sortie de ses propres citoyens vers un autre pays. Source d'instabilité mais aussi de création de richesse (apport de devises) et d'instrumentalisation politique (création de groupes de pression dans le pays d'expatriation), le fait migratoire a souvent fait l'objet de politiques publiques dans plusieurs pays du monde. Ainsi, la Turquie, comme d'autres, veille scrupuleusement à la nature de ses relations avec ses ressortissants, d'autant plus qu'elle a une certaine expérience en la matière. Depuis les années 1960, des millions de travailleurs turcs ont immigré en Allemagne et en France, créant une communauté dont le poids politico-religieux ne peut la laisser indifférente.

41 En Asie centrale et dans le Caucase, elle veille plus particulièrement à ce que la présence de ses ressortissants ne détériore pas ses relations avec les États de la région. Dans certains cas, elle cherche même, comme d'autres États le font ailleurs, à utiliser les personnes engagées voire embrigadées dans les actions d'associations ou de mouvements informels privés, pour les mettre au service de sa politiqué extérieure.

S'agissant des rapports avec les mouvements missionnaires turcs en Asie centrale et dans le Caucase, la République turque, séculière et laïque, maintient une attitude pour le moins pragmatique. Tandis que sur son territoire, les relations entre l'État et les organismes religieux peuvent être tendues, en Asie centrale, pour des raisons de convergence d'intérêts supérieurs, les relations entre l'action de l'État et celle des mouvements religieux privés sont bonnes voire complémentaires. Dans des pays où la société civile et les acteurs non étatiques restent pour le moins inexistants, les régimes éprouvent souvent le besoin de demander la garantie, la caution de l'État turc et de ses ambassades pour entamer un quelconque projet de coopération avec ces organisations turques. Le cas le plus représentatif est l'exemple de Fethullah Gülen et de son vaste 
réseau éducatif. Malgré les mauvaises relations que le mouvement entretient avec l'État en Turquie, les ambassades turques en Asie centrale ont toujours apporté leur soutien aux disciples de Gülen qui ont ainsi pu plus facilement asseoir un vaste réseau d'écoles privées appuyées par des entreprises turques, elles-mêmes gérées par des expatriés turcs aux motivations religieuses affirmées.

L'implantation des mouvements islamistes turcs en Asie centrale par le biais des migrants pose la question du caractère provisoire ou définitif de cette implantation des idées et des hommes. La plupart des migrants turcs en Asie centrale resteront sans doute dans leur pays d'accueil car on voit déjà se multiplier les accessions à la propriété mais surtout les mariages mixtes qui sont le meilleur indicateur du caractère définitif d'une immigration. Pour ce qui est de la prégnance des idées religieuses importées de Turquie, il convient d'être plus nuancé. En effet, leur enracinement en Asie centrale ne pourrait se faire sans concession aux pratiques et coutumes locales. Ainsi, une sorte de syncrétisme musulman turc et centrasiatique, très proche au demeurant, s'élabore chaque jour, au fur et à mesure que les immigrés anatoliens prennent leurs marques dans les pays d'origine de leurs illustres aïeuls.

Quant au rêve de grandeur de la Turquie et son relent d'empire avec l'immigration de ses ressortissants en Asie centrale, il s'agit là d'une question complexe, dont la réponse ne peut être que partielle et temporaire. La présence des entreprises, écoles, mouvements religieux mais aussi simples travailleurs turcs en Asie centrale a incontestablement accompagné et même aidé la Turquie à y asseoir une certaine influence. Toutefois, cet apport ne doit pas être exagéré car, en réalité, après avoir beaucoup misé sur l'Asie centrale et les retrouvailles avec les frères turcs, la Turquie est revenue à une politique plus réaliste, recentrée sur les vrais enjeux géopolitiques et stratégiques extérieurs, parmi lesquels l'Asie centrale ne figure pas. L'aventure centrasiatique, pétrie des illusions de retour dans la patrie originelle et mythique des Turcs, aura fait long feu et démontre que la Turquie regarde droit devant elle, vers l'Europe, et se détourne de son passé asiatique. Farouchement euro-centrée, c'est sur les millions d'immigrés turcs citoyens de l'Union européenne que la Turquie semble s'appuyer pour forcer la forteresse européenne où se concentrent ses intérêts les plus vitaux.

\section{NOTES}

1. Jacob LANDAU, Pan-Turkism : From Irredentism to Cooperation, Bloomington, Indiana University Press, 1995, $275 \mathrm{p}$.

2. Lowell BEZAINIS, "Soviet Muslim émigrés in the epublic of Turkey», Central Asian Survey, vol. 13, n 1, 1994 : 59-180.

3. Voir à ce titre, par exemple, Anthony HyMAN, " Turkestan and Panturquism revisited », Central Asian Survey, vol. 16, n 3, 1997 : 25. Voir également Bayram BALCY, La Turquie en Asie Centrale, la conversion au réalisme, Istanbul, IFEA (Dossiers de l'Institut français d'études anatoliennes ; 5), 2001, $110 \mathrm{p}$. 
4. Lerna YANIK, «The politics of education exchange : Turkish education in Eurasia », Europe-Asia Studies, vol. 56, n² 2, $2004: 293-302$.

5. Lowell BEZAINIS, op.cit.

6. Lerna YANIK, op.cit.

7. Voir sur cette question de gestion des indépendances, Marlène LARUELLE et Sébastien PEYROUSE, "Gestion de l'indépendance et legs soviétiques en Asie centrale", Les Cahiers d'Asie centrale, $n^{\circ} 13-14,2004: 9-18$.

8. Colin TURnER, Hasan HorkUC, Said Nursi : Makers of Islamic Civilization, Oxford, IB Tauris, 2009, $160 \mathrm{p}$.

9. Adil SonmEZ, Fethullah Gülen ve Vizyonu (Fethullah Gülen et sa vision du monde), Istanbul, Kaynak Yayinlari, 1998, 438 p.

10. Bayram BALCI, Les missionnaires de l'Islam en Asie centrale. Les écoles turques de Fethullah Gülen, Paris, Maisonneuve et Larose, 2003, 301 p.

11. Bayram BALCI, Les missionnaires de l'Islam en Asie Centrale..., op.cit.

12. Voir le site des disciples de Suleyman Tunahan, http://www.tunahan.org, site consulté à la date du 29 octobre 2010.

13. Sur la Nakshibendiyya et autres grandes confréries originaires d'Asie centrale, voir Alexandre Popovic, Gilles VeInSTEIN, Les voies d'Allah. Les ordres mystiques dans le monde musulman des origines à aujourd'hui, Paris, Fayard, 1996, $711 \mathrm{p}$.

14. Thierry ZARCONE, "Ahmad Yasavi, héros des nouvelles républiques centrasiatiques ", Revue des Mondes musulmans et de la Méditerranée, n89-90, 2000 : 297-323.

15. Sur le groupe confrérique nakshibendi d'Osman Nuri Topbas, voir les sites de la mouvance: http://www.gonuldunyamiz.com.. Site consulté à la date du 29 octobre 2010.

16. Mushfig B AYRAM, "UZBEKISTAN: Roadblocks around trial, more Nursi readers arrested", Forum 18, 10 May 2010, http://www.forum18.org/Archive.php?article_id=1442 (dernière consultation à la date du 29 octobre 2010).

\section{RÉSUMÉS}

Forte de ses liens de parenté culturelle avec les Républiques turcophones d'Asie centrale Kazakhstan, Kirghizstan, Ouzbékistan, Turkménistan et Tadjikistan -, la Turquie a été le point départ d'un important mouvement migratoire en direction de ces États à partir du début de la décennie 1990, au moment où les États en question recouvraient leur indépendance survenue avec la fin de l'Union soviétique. Multiforme, cette migration a véhiculé un important message religieux, dans les deux sens, mais surtout en provenance de Turquie et à destination des principales villes d'Asie centrale. C'est le principal sujet d'investigation de cette étude. Le commerce et la coopération éducative furent les principaux outils déployés par les migrants turcs partis souvent en tant que missionnaires pour ré-islamiser l'Asie centrale, que de nombreux mouvements islamiques turcs jugeaient devoir être remise dans le giron de la civilisation islamique après plus de soixante-dix ans d'une domination soviétique caractérisée par une politique antireligieuse prononcée. Quatre principaux mouvements islamiques turcs se sont fait remarquer en Asie centrale dans cette œuvre de prosélytisme. Le plus important est sans conteste celui fondé par Sait Nursi dont les disciples ont été très actifs dès 1990 dans l'envoi de littérature islamique en Asie centrale. Par ailleurs, un disciple de Sait Nursi, Fethullah Gülen, a 
fondé un vaste réseau éducatif animé par des jeunes éducateurs partis d'Anatolie pour s'investir dans différentes villes d'Asie centrale. Une autre mouvance, dite suleymanci, du nom de son fondateur Suleyman Tunahan, a, par le biais de la migration turque, ouvert plusieurs petites madrasas dans divers États d'Asie centrale. Enfin, des disciples de la confrérie dite nakshibendiyya, du nom de son fondateur Bahaduddin Nakshibend, un mystique $d u \mathrm{xv}^{\mathrm{e}}$ siècle originaire de Boukhara, ont également envoyé des milliers de migrants turcs dans le cadre de plusieurs projets missionnaires. Amenée à s'enraciner dans ses villes d'expatriation en Asie centrale, cette migration turque a déjà très nettement marqué de son empreinte l'islam de ces pays, plus particulièrement au Turkménistan, au Kazakhstan et au Kirghizstan qui ont d'excellentes relations politiques avec la Turquie. Ainsi, bon nombre de nouvelles élites religieuses dans ces pays ont été formées dans le cadre de la coopération avec les mouvements missionnaires turcs et leurs disciples qui ont immigré en Asie centrale. S'inscrivant en marge de la politique officielle turque de coopération en matière religieuse avec les pays d'Asie centrale, les migrants turcs n'en rendent pas moins un grand service à la diplomatie d'Ankara dans la région en l'aidant indirectement à se constituer une sphère d'influence dans cet espace turcophone qui occupe une place notable dans la nouvelle politique extérieure de la Turquie.

With the fall of the Soviet Union in the early 1990s, a wave of migrants left Turkey for the newly independent Turkish-speaking Central Asian republics with which the country had strong cultural ties - Kazakhstan, Kyrgyzstan, Uzbekistan, Turkmenistan and Tajikistan. As a result of this multiform movement, an important religious message travelled in both directions, but especially from Turkey to the main Central Asian cities. This article focuses on this message. Turkish migrants, who were often missionaries, used commerce and cooperation initiatives in education as two main tools to re-Islamise Central Asia. For many Turkish Islamic movements, it was important to bring this region back into the fold after over 70 years of domination by a Soviet government with a strongly anti-religious policy. As part of this proselytizing, four key Turkish Islamic movements became visible in Central Asia. The largest was undoubtedly that founded by Said Nursi, whose disciples were active in sending Islamic literature to Central Asia from 1990. One of Said Nursi's disciples, Fethullah Gülen, founded an extensive educational network, involving young instructors from Anatolia who moved to different Central Asian cities. A second group, the Suleymanci, named for its founder Suleyman Tunahan, used Turkish migration to open several small madrasas in different Central Asian countries. Finally, disciples of the Naqshbandiyya brotherhood, named after its founder Bahauddin Naqshband, a $15^{\text {th }}$ century mystic from Bukhara, also sent thousands of Turkish migrants to the region to carry out missionary projects. After settling in these Central Asian cities, Turkish migrants have had a significant effect on Islam in the region. This is especially true in Turkmenistan, Kazakhstan and Kyrgyzstan, which have excellent political relationships with Turkey. As a result, many of these countries' new religious elites have formed as a result of cooperation with Turkish missionary movements and their disciples who immigrated to Central Asia. Turkish migrants are not part of Turkey's official policy on religious cooperation with Central Asia. However, they support Ankara's diplomatic efforts in the region by indirectly creating a field of influence in this Turkish-speaking region, which is of considerable importance in Turkey's new foreign policy. 
INDEX

Thèmes : islamisation, migration, mobilité

Mots-clés : éducation, islamisation, mouvance suleymanchi, nakshibendiyya, Sait Nursi

Index géographique : Asie centrale, Kazakhstan, Kirghizstan, la Turquie, Ouzbékistan,

Turkménistan et Tadjikistan

Keywords : Central Asia, education, islamization, Kazakhstan, Kyrgyzstan, nakshibendiyya, Sait Nursi, suleymanchi movement, Turkey, Turkmenistan and Tajikistan, Uzbekistan

\section{AUTEUR}

BAYRAM BALCI

Diplômé en science politique et en études islamiques à l'Institut d'études politiques de Grenoble et d'Aix-en-Provence, il a travaillé plusieurs années dans le Caucase et en Asie centrale. Ancien directeur de l'Institut français d'études sur l'Asie centrale (IFEAC-UMIFRE 10) basé à Tachkent, ses recherches concernent essentiellement le renouveau islamique et les problématiques migratoires dans toute l'ex-Urss.

balci_bayram@yahoo.fr 